
\title{
R Reseacrch Surare \\ Cost-efficient multiplex PCR-based metabarcoding with new comprehensive primer sets for monitoring plankton diversity dynamics
}

Shanmei Zou ( $\nabla$ zousm912@njau.edu.cn )

Nanjing Agricultural University https://orcid.org/0000-0002-5253-3314

Lydia Smith

UC Berkeley: University of California Berkeley

\section{Research}

Keywords: Metabarcoding, Plankton, New primer sets, Multiplex-PCR, Next Generation Sequencing

Posted Date: January 20th, 2022

DOI: https://doi.org/10.21203/rs.3.rs-97365/v2

License: (c) (i) This work is licensed under a Creative Commons Attribution 4.0 International License. Read Full License 


\section{Abstract}

Aim: Accurate and quick monitoring of plankton diversity dynamics is critical and urgent for aquatic environmental protection, especially for bloom management. Multiple genetic markers with efficient primers should be considered for accurate species detection by metabarcoding. Here we aim to provide cost-efficient multiplex PCR-based metabarcoding (CMPM) with new comprehensive primer sets for monitoring plankton diversity dynamics. The CMPM assay was tested in samples from Lake Tai.

Location: Freshwater Lake Tai, China

Methods: A total of nine primer sets from 18S, 28S, 23S, 16S and $r b c L$ was presented, including five newly designed ones 18S-v4-5, 28S, 16S-new, rbcL-Chlo and rbcL-Cry in this study. Three multiplex PCR-based system were developed for Next Generation Sequencing of all nine genetic markers. The assignment efficiency of new primer sets for plankton were detected. The taxonomic assignments of all genetic markers were compared in each of species, genus and family level. The single and multiplex PCR-based metabarcoding were compared. The assignments from multiple genetic markers and microscope observations were compared in the reference samples. The CMPM assay was tested in samples from Lake Tai.

Results: The taxonomic assignments of all nine genetic markers differed from each other in each of species, genus, family and phylum level. The $28 \mathrm{~S}$ identified a higher proportion of phytoplankton, zooplankton and fungi with high assignment performance in species level. The 16S-new marker not only obtained large cyanobacteria assignments, but also obtained other assignments from phytoplankton and bacteria. The new 18S-v4-5 was much variant to detect a broader range of zooplankton and phytoplankton. The two new primer sets of $r b c L$-Chlo and $r b c L$-Cry specifically assigned Chlorophyta and Cryptophyta successfully. The multiple genetic markers produced more plankton assignments than microscope observations. The plankton communities in Lake Tai showed pronounced seasonal dynamic by CMPM, which is consistent with the previous reports.

Main conclusions: The 28S, 16S-new and 18S-v4-5 newlywed designed here could be as complementary universal genetic markers for identifying plankton. The $r b c L-C h l o$ and $r b c L-C r y$ could be as specific markers for identifying Chlorophyta and Cryptophyta respectively. The CMPM assay can provide precise identification and save cost for monitoring plankton diversity dynamics with comprehensive primer sets.

\section{Introduction}

It is a big challenge for biologists to quantify environmental biodiversity just relying on morphological and behavioral characters (Burivalova et al. 2019; Outhwaite et al. 2020; Beng et al. 2020). Next Generation Sequencing (NGS) is becoming an effective tool for biodiversity monitoring in a wide range of environmental samples by overcoming some of the challenges of morphological identification, which makes the environmental DNA (eDNA) metabarcoding fast for monitoring biodiversity without significant damage to the target species or its habitats (Eisenstein, 2018; Alberdi et al. 2018; Alexander et al. 2020; Takahara et al. 
2020). The metabarcoding can also increase the probability of detecting rare, cryptic and elusive species (Needham and Fuhrman, 2016; Pierre et al. 2018; Franklin et al. 2019; Shelton et al. 2019; Beng et al. 2020).

The challenges for metabarcoding include incomplete reference database and PCR primer bias. Assigning abundant NGS reads mainly relies on available reference sequences in public databases. However, most species just have one or a few genetic marker sequences available in public reference databases. In this case, some reads can only be assigned to higher taxonomic levels, which makes it difficult to associate eDNA data with existing biological and ecological knowledge (Beng et al. 2020). The methods of building reference database also affects the identification accuracy, like blasting against the NCBI database directly, downloading a local copy, or building a local reference (Pierre et al. 2018). The ecoPCR is able to obtain relatively complete and accurate reference sequences for one pair primers by in-silico PCR (Ficetola et al. 2010; Pierre et al. 2018; Emily et al. 2019). Secondly, successful amplification of molecular markers for metabarcding depends highly on primer specificity, sensitivity, and efficiency (Nichols et al. 2018). However, it is almost impossible to amplify all taxa expected successfully for one genetic marker. Then special primers need to be designed for specific taxonomic groups to get comprehensive taxonomic identification. Additionally, one short single gene usually provides insufficient information for identifying all species. Conserved genetic primers have to be designed to get compatible short fragments with sequencing platforms within a limited length (Bradley et al. 2016; Bahram et al. 2018; Knight et al. 2018). Thus, researchers suggest that multiple genetic markers with efficient primers should be considered for accurate species detection (Bucklin et al. 2016; Zhang et al. 2018).

Amplicon-based NGS library preparation is also critical in reducing PCR bias, which mainly differ in whether the sequence adaptors are added by ligation (Claesson et al. 2010; Degnan et al. 2012), single-step PCR (Bartram et al. 2011; Fadrosh et al. 2014) or multi-step PCR (Lundberg et al. 2013). A two-step PCR library preparation is recommended since it can avoid potential bias due to the use of different indices in each primary amplification and offers increased versatility by minimizing the number of primers that must be synthesized. While multiple genetic barcode primers are employed for metabarcoding, the cost of library preparation should also be considered due to multifold cost in PCR, cleanup and DNA quantification, especially for large samples. Multiplex PCR is a widespread molecular biology technique for amplification of multiple genetic targets in a single PCR experiment, which could be as a cost-effective method for metabarcoding if we address the challenges of suitable primers used, effective PCR conditions, and good library preparation (Henrik et al. 2018).

The water ecosystem has gradually been altered by climate change, increasing nutrient pollution and activities of humans (Drake, 2014; Baldwin et al. 2014), which often lead to eutrophication and algae blooms (Jef et al. 2018). Cyanobacterial blooms are currently increasing globally (Jef et al. 2018). Actually, not only the cyanobacteria bloom but also many other photosynthetic algae bloom, such as green algae and diatoms, are also serious (Needham and Fuhrman, 2016, Berdjeb et al. 2018). While phytoplankton metabarcoding in marine environment has been triggered with $16 \mathrm{~S}$ or 18S NGS (Needham and Fuhrman, 2016, Berdjeb et al. 2018) the phytoplankton metabarcoding in inland lakes with bloom is lagging behind, especially for cyanobacteria. Lake Tai is one of the most important water resources for drinking and development of agriculture and industry in China. However, the blooms in Lake Tai have occurred frequently since 1997, 
which brought ecosystems to the brink and led to serious environmental problems (Qin et al. 2010; Duan et al. 2015; Jef et al. 2018). Recent years the blooms often changed with seasons and gradually occurred among the whole lake (Zou et al. 2016a, Zou et al. 2016b; Zhang et al. 2018; Li et al. 2018).

Here we developed cost-efficient multiplex PCR-based metabarcoding (CMPM) assay with new multiple primer sets and tested them in samples from Lake Tai, aiming to:1) provide new comprehensive multiple genetic primer sets for plankton diversity monitoring. 2) construct cost-effective multiplex PCR-based assay for microbial metabarcoding. 3) test the CMPM assay for revealing diversity and spatio-temporal dynamics of plankton. Three multiplex PCR systems were performed to amplify nine genetic markers, including three regions of 18S (18S-v1-3, 18S-v4-5, 18S-v9), two regions of 16S rDNA, 23S rDNA, 28S rDNA and two regions of $r b c L$ (Figure 1, Table 1). The 18S-v4-5, 28S, 16S-new, $r b c L$-Chlo and $r b c L$-Cry were newly designed in this study.

\section{Methods}

\section{Sample collection and DNA extraction}

The whole Lake Tai was divided into five regions based on the reported plankton distribution experience for spatio-temporal dynamics evaluation (Figure S1). The samples were collected by use of a plankton net $(0.2 \mu \mathrm{m})$ through which $30 \mathrm{~L}$ of water were passed. Mixed samples were collected at each sampling site by combining an equal volume of water samples collected from $0.5 \mathrm{~m}$ depth, $1.0 \mathrm{~m}$ depth and $2.0 \mathrm{~m}$ depth. The 20-25 samples were collected from each of five regions on April 1 to 3 and August 1 to 3 in 2017 (Table S1). Thereinto, a total of 40 samples from April and August (20 for April and 20 for August respectively) were used as reference samples identified by microscope observations, where the phytoplankton were identified by microscope for comparing species assignments of all primer sets. For each of the 40 reference samples, all of nine genetic primer sets from three PCR systems in Table 1 were amplified. The rest 152 samples were considered as non-reference samples, which were just amplified by PCR system A in Table 1. The PCR amplification of all samples were triple.

DNA was extracted by OMEGA E.Z.N.ATM Mag-Bind Soil DNA Kit for each sample. The water temperature (WD), total phosphorous (TP), total nitrogen (TN), ammonia nitrogen ( $\left.\mathrm{NH}^{+}-\mathrm{N}\right)$, and nitrate nitrogen (NO3- $\left.-\mathrm{N}\right)$ were detected and analyzed based on a standard method common used (Li et al. 2018). The detailed environmental factor parameter for all samples were showed in Table S1.

\section{Primer selection and new primer design}

Firstly, multiple genetic primers that have previously been proved useful were selected from published papers. We aimed to select multiple genetic primers which were useful to identify a wide range of phytoplankton and zooplankton. The length of the target should be below $500 \mathrm{bp}$ for compatibility with the maximum read length of the Illumina system. Based on these criteria, about 50 published pairs of locusspecific primers were selected and tested initially. Finally, 18S-v1-3, 18S-v9, 23S and 16S which already have available published primers were selected (Table 1). Due to PCR failure for our samples from published 18S- 
v4 primers, new 18S-v4 primers were designed. New $28 \mathrm{~S}$ primers were also designed. We also designed two primer sets of $r b c L$, which could specifically identify Chlorophyta and Cryptophyta.

The related reference sequences for primer design were downloaded from EMBL database (8/16/2019). The $18 \mathrm{~S}$ reference database mainly includes the complete $18 \mathrm{~S}$ sequences. The $28 \mathrm{~S}$ reference database includes the eukaryotic $28 \mathrm{~S}$ sequences. The $16 \mathrm{~S}$ reference database includes the prokaryotic and eukaryotic $16 \mathrm{~S}$ sequences. The two $r b c L$ references databases just include Chlorophyta and Cryptophyta sequences respectively. The program DegePrime was used to design new primers (Hugerth et al. 2014), which uses weighted randomized combination to find the degenerate primer with highest coverage for every window of a sequence alignment. Firstly, the related reference sequences were aligned by FAMSA (Deorowicz et al. 2016). The TrimAlignment was then used to remove columns scarce in aligned sequences since the sequence alignments can include many gaps. Then the DegePrime was conducted to produce a primer list which showed the sequence coverage of each primer. Finally, we selected primers with higher coverage to further test by PCR. The new designed primers that were proved effective were chosen for next multiplex PCR.

\section{Multiplex PCR-based eDNA amplification and library preparation}

Firstly, we amplified each genetic locus individually using a Qiagen Hot-Start PCR kit to confirm that they will produce only one single clear fragment, where PCR reactions were carried out in a total volume of $10 \mu \mathrm{L}$. Then multiple pairs of locus-specific primers were mixed together for gradient PCR (annealing temperatures from $45^{\circ} \mathrm{C}$ to $68^{\circ} \mathrm{C}$ ) to select optimal multiplex PCR conditions under which multiple genetic loci could be amplified together without cross-reactions. Finally, three PCR systems from the 9 primer sets could be amplified in one PCR reaction respectively with different annealing temperatures (Table 1). Multiplex PCR reactions were carried out in a total volume of $25 \mu \mathrm{L}$ with Qiagen Multiple PCR Plus kit, using $12.5 \mu \mathrm{L}$ Master Mix, $1 \mu \mathrm{L}$ DNA template $(2-10 \mathrm{ng} / \mu \mathrm{L}), 0.1 \mu \mathrm{M}$ of forward and reverse primer, and Q-Solution. PCR conditions for the three multiplex PCR systems were: $95^{\circ} \mathrm{C}$ for 5 min, 30 cycles of $95^{\circ} \mathrm{C}$ for 30 s, primer-specific annealing temperatures for $1 \mathrm{~min} 30 \mathrm{~s}, 72^{\circ} \mathrm{C}$ for $1 \mathrm{~min} 30 \mathrm{~s}$, with a final extension of $72{ }^{\circ} \mathrm{C}$ for $1 \mathrm{~min}$. Then two-step NGS library preparation was performed. Primers were synthesized with the locus-specific sequence on the $3^{\prime}$ end and a $5^{\prime}$ tail containing sequences matching the TruSeq sequencing primer binding site. The forward primer was synthesized with a $5^{\prime}$ tail of ACACTCTTTCCCTACACGACGCTCTTCCGATCT and the reverse primer with a 5 ' tail of GTGACTGGAGTTCAGACGTGTGCTCTTCCGATCT. The first PCR was conducted with these tailed primers using annealing temperatures $55^{\circ} \mathrm{C}$ for PCR system $\mathrm{A}$ and $\mathrm{B}$, and $49^{\circ} \mathrm{C}$ for PCR system C (Figure 1). Triple PCR products for each sample were pooled, which was then cleaned by Solid Phase Reversible Immobilization (SPRI) Beads. After purification and quantification, equal amounts of PCR products from the three multiplex PCR systems for each sample were pooled. Then, indexing PCRs were performed for each pooled amplicon with indexing primers annealing to 5 '-tails of the locus-specific PCR primers. The indexing primers include dual indexes and Illumina sequencing adapters provided by Vincent Coates DNA Sequencing Laboratory at UC. Finally, all the amplicons were pooled in equal amounts for sequencing on an Illumina MiSeq 600 cycles. The whole workflow was shown in Figure 1. For comparing the assignment consistency between singleplex and multiplex PCR, we selected 6 samples to perform the single PCR for each locus per specimen, where the PCR conditions were the same as multiplex PCR. Finally the assignment rate in species, genus and family level from each genetic marker was performed for 
Cyanobacteria, Bacillariophyta, Chlorophyta, Cryptophyta and Zooplankton respectively. Here the assignment rate means the ratio of species, genera and families assigned among all the assignments for each taxonomic group from each marker.

\section{Bioinformatics analysis}

The ecoPCR (Ficetola et al. 2010) was firstly conducted to build the original reference database for each genetic marker on the EMBL standard nucleotide database

(ftp://ftp.ebi.ac.uk/pub/databases/embl/release/) (Stoesser et al. 2002) (September, 2019). For each genetic locus, 3 mismatches was set for in-silico PCR. The blastn (Camacho et al. 2009) was then used to query the seed databases against the NCBI non-redundant nucleotide database (Pruitt et al. 2005) for increasing the breadth of reference sequences and capturing sequences without barcode primers in EMBL, as modified from CRUX module in Emily et al. (2019). Taxonomy files were retrieved using Entrez-qiime (Baker, 2016). We also added our previous published phytoplankton sequences from traditional DNA barcoding into the reference databases for $16 \mathrm{~S}$ and $r b c L$. Finally, if there were still reads which could not be assigned to any taxa we blasted them against the whole NCBI nucleotide database again to get the unique perfect-matched species assignment.

Sequence analysis for all genetic markers was performed using QIIME2 (Bolyen et al. 2019). Raw sequence fastq files were firstly demultiplexed to separate the samples based on the indexes. PCR primers were removed by cutadapt package in QIIME2. Dada2 in QIIME2 was applied for denoising, dereplicate-sequence, filtering chimera and merging the paired reads. For 18S-v1-3, 18S-v4-5 and 16S-new, we selected paired reads merged with good quality score or only forward reads (if the paired reads could not be perfectly jointed) for next assignment analysis. After quality filter, taxonomic classification was performed by feature-classifier module with Naive Bayes. For reads that were identified as unsigned by QIIME2 we blasted them against the whole NCBI (nt/nr) database to get their unique perfect-matched species assignment with the highest identity score 1 . Finally, the taxonomic assignments and their reads abundance from all genetic loci were obtained for biodiversity analysis. The taxonomic assignments from reference samples selected were used to compare the identification efficiency of all genetic loci. The proportion of taxonomic assignments at the phylum level was analyzed firstly. Then at each of species, genus and family level within each phytoplankton phylum, the taxonomic dissimilarity of all genetic loci was compared by Venn package in $\mathrm{R}$

(https://www.rstudio.com/products/rpackages/). Here special venn diagram with column bars was used for more than 7 elements compared, which was conducted by upset plots. For each phylum, among all primer sets, only primers that had success PCR amplification and sequencing were included for analysis. The assignment consistency between the single PCR and the multiplex PCR in the species level was compared. The final assignments for each genetic marker include taxonomic level in species, genus, family, order, etc., including some un-assignments. For understanding the identification effectiveness in each taxonomic level for each genetic marker, we conducted the assignment statistics in species, genus and family level respectively, where we calculated the ratio that the amount of species, genera and family assigned takes up the total assignments respectively. 
The taxonomic assignments of both reference and non-reference samples from all genetic loci were used for diversity and dynamics evaluation. The top taxa of phytoplankton and zooplankton from each season was indicated by heatmap implemented in $\mathrm{R}$ package. For spatio-temporal dynamics analysis, pairwise community dissimilarity was calculated using Bray-Curtis as implemented in the vegan package (Oksanen et al. 2019). The non-metric multidimensional scaling (NMDS) plot based on Bray-Curtis distance was generated to reveal the spatio-temporal dynamics by vegan. The Canonical Correspondence Analysis (CCA) analysis was performed to evaluate the correlations of environmental factors and spatio-temporal dynamics, with the "cca" function from vegan package (Oksanen et al. 2019). The reference and non-reference samples were analyzed independently to avoid sample bias. The vegan package was employed in R 3.6.1. The detailed environmental factor parameters for all samples from two seasons were listed in Table $\mathrm{S} 1$.

\section{Results}

\section{Sequence coverage for the new primer sets}

Five gene regions of $r b c L, 28 \mathrm{~S}, 18 \mathrm{~S}$ and $16 \mathrm{~S}$ were newly designed using the program DegePrime, including $28 \mathrm{~S}, 18 \mathrm{~S}$-v4-5, 16S-new, $r b c L$-Chlo and $r b c L$-Cry (Figure 2). The program DegePrime can present us the conserved and variant regions among the whole alignment reference sequences and thus found the most conserved regions for primer selection. From the primer coverage plot it was indicated that the $28 \mathrm{~S}$ sequences are much variant among the whole assignment positions with high primer coverage, e.g. between 200-600 (Figure 2). The sequences around the position 600 of $28 \mathrm{~S}$ are much conservative with coverage rate more than $90 \%$, which was thus selected as the reverse primer. The sequences around the position 200 of $28 \mathrm{~S}$ are also relatively conservative, which was selected as the forward primer. This pair primers were proved effective for PCR the plankton groups (Figure 3). The 18S was apparently conservative among the whole alignment positions, where the $18 \mathrm{~Sv} 4$ region was relatively variant. Thus we selected the conserved forward and reverse primers from the two ends of $18 \mathrm{~Sv} 4$ and $18 \mathrm{~Sv} 5$ regions to amplify the variant regions. For $r b c L-$ Chlo and 16S-new, the primer coverage is relatively low, which means that the both genetic markers are highly variant among all alignment positions without much conserved primers. So we selected the primers which has possibility to amplify more species. The $r b c L$-Cry gene has generally high primer coverage for almost all alignment positions.

\section{Comparison of taxonomic assignments for all primer sets}

After filtering reads with low identify score the "clean reads" for samples of April and August were 4,348,278 and 4,816,816 respectively, which were assigned to cyanobacteria, eukaryotic phytoplankton, zooplankton, fungi, few bacteria and some unassigned taxa.

The proportion of various taxonomic assignments from each gene barcode was shown in Figure 3. Among the genetic primer sets, the $18 \mathrm{~S}-\mathrm{v} 4-5$ and the new designed marker $28 \mathrm{~S}$ assigned relatively even proportion for various eukaryotic groups of Chlorophyta, Bacillariophyta, Zooplankton, Fungi and Cryptophyta, together with a small proportion for Chrysophyta, Pyrrhophyta and Ochrophyta. Both 18S-v1-3 and 18S-v9 can also assign Chlorophyta, fungi and Pyrrophyta, but they assigned a high proportion for zooplankton. The $23 \mathrm{~S}$ and $16 S$ obtained a higher proportion of cyanobacteria assignments, with a small proportion of Bacillariophyta, 
Cryptophyta, Chrysophyta, Euglenida and Pyrrophyta. The new designed 16S-new marker not only obtained cyanobacteria assignments, but also obtained other assignments from Bacillariophyta, Chlorophyta, Chrysophyta, Cryptophyta, Pyrrophyta and bacteria. Thus the 16S-new could be as universal genetic marker for identifying various taxa of phytoplankton. We also designed specific primers for amplifying Cryptophyta (rbcL-Cry) and Chlorophyta (rbcL-Chlo), which assigned a high proportion of Cryptophyta and Chlorophyta respectively. However, there was a proportion of reads that could not be assigned to any taxonomic level, being considered as unassigned taxa.

Based on the assignments from the 48 reference samples, the taxonomic difference of all genetic primer sets at each of species, genus and family level was shown in Figure 5 and Figure S2. The 28S produced the most unique Bacillariophyta assignments from other genetic primers at each of species, genus and family level (Figure 5). The 18S-v4-5 produced the second most unique Bacillariophyta species assignments from other genetic markers (Figure 5). For Chlorophyta, while the 18S-v4-5 produced the most unique species assignments, the $28 \mathrm{~S}$ produced the most unique genus assignments (Figure 5). The 16S-new obtained the most unique cyanobacteria assignments at each of species, genus and family level (Figure 5). For zooplankton, the most unique assignments were from 18S-v4-5 at each of species, genus and family level (Figure 5). The 18S-v4-5 also obtained the most unique species assignments for Chrysophyta and Cryptophyta (Figure S2). The $28 \mathrm{~S}$ obtained the most unique genus assignments for Cryptophyta. Finally, the phytoplankton species assignments from all genetic primers were compared with the microscope observations (Figure 6). For each phylum, while some gene barcodes showed the same species assignments with the microscope observations, other gene barcodes showed unique species assignments from microscope observations. For Bacillariophyta and Cyanobacteria, the microscope observations showed some different species assignments from all gene barcodes. The detailed species list of multiple gene barcodes and microscope observations for each phytoplankton phylum was listed in Table S2-Table S8.

\section{Identification efficiency of each primer set in each of species, genus and family level}

We conducted the statistics for assignment rate at species, genus and family level from each genetic marker for Cyanobacteria, Bacillariophyta, Chlorophyta, Cryptophyta and Zooplankton respectively. All the genetic makers showed relatively lower assignment rate in species level than that in genus and family level (Figure 4). The 235 performed well for identifying Cyanobacteria in species level (more than $80 \%$ assignment rate), and performed well in genus and family assignments for both of Cyanobacteria and Bacillariophyta. The $28 \mathrm{~S}$ had high assignment rate in each of species, genus and family level for all of Bacillariophyta, Chlorophyta, Cryptophyta and Zooplankton (more than 68\%), especially for Bacillariophyta and Cryptophyta. For all of Bacillariophyta, Chlorophyta, Cryptophyta and Zooplankton, both 18S-v9 and 18S-v4-5 produced relatively low assignment rate in species level, but higher assignment rate in genus and family level.

Apparently the 18S-v1-3 produced weak assignments in species level for both Chlorophyta and Zooplankton. The universal $16 \mathrm{~S}$ marker had low species assignment rate but high genus and family assignment rate for Cyanobacteria and Bacillariophyta. The 16S-new showed high assignment rate in species level for Cyanobacteria and Cryptophyta. The rbcL-Cry and rbcL-Chlo showed moderate assignment rate in species level for Chlorophyta and Cryptophyta.

\section{Comparison of singleplex and multiplex PCR-based metabarcoding}


The assignment consistency of singleplex and multiplex PCR was confirmed by observing the ratio of their shared assignments to their total assignments. It was shown that there was assignment dissimilarity in species level between singleplex PCR and multiplex PCR for most of the genetic primers from 6 reference samples (c1, c2, c3, c4, c5, c6) (Figure 7). The consistency rate (the proportion of shared assignments to their total assignments) was generally lower than 0.8 for all genetic markers for samples c1-c5. In sample c6, the assignments of singleplex PCR and multiplex PCR from $r b c L$-Chlo and $r b c L$-Cry were generally consistent with near $100 \%$ consistency rate. We also found that some genetic markers in the multiplex PCR systems amplified more taxa than that in the singleplex PCR system. But some genetic markers in the singleplex PCR system also amplified different taxa from the multiplex PCR system. There was cross amplification between singleplex and multiplex PCR reactions.

\section{Use of CMPM for plankton diversity dynamics in Lake Tai}

The proportion of various taxonomic assignments was generally consistent between April and August in Lake Tai, where the cyanobacteria made up a large proportion followed by zooplankton, Chlorophyta, Bacillariophyta and Cryptophyta (Figure 8A). The top species and genera of phytoplankton from the two seasons were shown in Figure 8B and 8C. In genus level, the top 30 genera between April and August were different (Figure $8 \mathrm{C}$ ). The top 30 phytoplankton families for the two seasons were shown in Figure S3A, and the top 30 zooplankton species for the two seasons were shown in Figure S3B. The taxonomic assignments from all genetic primers were combined for NMDS and CCA analysis. For both reference and non-reference samples, it was demonstrated that the samples in April and August were separated clearly for each of Bacillariophyta, Chlorophyta, Cryptophyta, Fungi and Zooplankton (Figure 9). For Cyanobacteria, Chrysophyta and Euglenida, the samples from the two seasons were also separated clearly. The separation of reference and non-reference samples was due to their different assignments from different gene barcodes. The samples for Pyrrophyta from the two seasons were not clearly separated despite some diversity dissimilarities, which was possibly because that the reads abundance was insufficient for clustering. The CCA analysis was further conducted for each phytoplankton phylum, zooplankton, and fungus respectively. The reference and non-reference samples were analyzed separately to avoid sample bias (Figure 10, Figure S4). It was shown that the temperature (WD) was remarkable in affecting community diversity variation among the whole lake from April to August with the longest vector, where the samples in the two seasons were separately clearly (Figure 10, Figure S4).

\section{Discussion}

Multiple primer sets would greatly contribute to the accuracy of microbial diversity monitoring for ecological study (Bucklin et al. 2016; Zhang et al. 2018). However, the universal 18 S primers can only identify part taxa of aquatic phytoplankton and zooplankton due to primer incompatibility. Particularly for phytoplankton, the $r b c L$ and 23S seem more effective for identification (Zou et al. 2016 a,b; Sherwood et al. 2007). In this study, we designed multiple sets of specific primers for metabarcoding plankton with cost-effective library preparation profile for NGS sequencing, which was then tested in Lake samples.

Firstly we compared the identification efficiency of multiple primer sets at each of species, genus, family and phylum level. It is clear that the 9 primer sets produced different assignments from each other, especially for 
the new primer sets. The $23 \mathrm{~S}$ and the $16 \mathrm{~S}$-new genes can be effective barcodes for detecting cyanobacteria, which produce unique cyanobacteria assignments from each other and have high assignment rate in species level. Particularly for the 16S-new marker, it not only can assign the bacteria but also can assign the cyanobacteria, Bacillariophyta, Chlorophyta, Chrysophyta, Cryptophyta, Pyrrophyta. Thus the 16S-new could be as good universal genetic marker for metabarcoding plankton. The universal $16 \mathrm{~S}$ primers can also identify most cyanobacteria, but its species assignment rate is low. The $28 \mathrm{~S}$ can be as another universal marker for monitoring eukaryotic phytoplankton and zooplankton with high identification ability in species level. For three regions of 18 S, the new 18S-v4-5 assigned relatively even proportion for Chlorophyta, Bacillariophyta and zooplankton, and produced different taxonomic assignments from 18S-v1-3 and 18S-v9 at each of species, genus and family level. We had tried to use available published $18 \mathrm{~S}-\mathrm{v} 4$ primers to amplify our samples (Berdjeb et al. 2018), but they failed to PCR the samples successfully. It is demonstrated that the 18S-v4-5 region newly designed in this study is more variable for identification of eukaryotic phytoplankton and zooplankton as previous reports (Bradley et al. 2016). The 18S-v9 and 18S-v1-3 are more effective for zooplankton identification. We also designed two $r b c L$ primer sets which specifically amplified Chlorophyta and Cryptophyta successfully and produced high-resolution in identifying Chlorophyta and Cryptophyta. Thus, we prove that it is critical to combine multiple genetic markers to get comprehensive taxonomic diversity of phytoplankton and zooplankton. Finally, the microscope identification was compared with the taxonomic assignments of each genetic marker for each phytoplankton phylum. The microscope observation identified some Bacillariophyta and Cyanobacteria species which were not detected by multiple genetic markers, which was because that there was no reference sequences for these species in public databases. We combined ecoPCR and blastn to get relatively complete reference database. But there were still some sequences which could not be assigned to any taxa due to the incomprehensive database. So it would be important to supplement as many as gene sequences into the public database (Karst et al. 2018). On the other hand, the multiple genetic markers assigned many species that the microscope observations did not identified. The species unidentified by microscope are possibly cryptic species or species that are difficult to be distinguished by morphological characters (Zou et al. 2016a,b).

While multiple genetic primers are used in high-throughput amplicon sequencing, the cost of amplicon library preparation is also multifold in target PCR, DNA cleanup, PCR enrichments, DNA quantity and index ligation, especially for large samples. The laboratory work is also time-consuming for multiple gene loci. Multiplex PCR is similar to the singleplex PCR except that each sample is designed to amplify and detect multiple target sequences rather than only a single target, which makes it possible to prepare the multiple amplicon NGS libraries once while the multiple targets are amplified together. However, multiplex methods must deal with interactions between multiple sets of primers that may cross-react. Henrik et al. (2018) firstly employed multiplex PCR in arthropod phylogenetic analysis to show the efficiency of multiplex PCR-based amplicon Illumina sequencing. Here we developed multiplex PCR-based amplicon Illumina sequencing for environmental biodiversity monitoring. Firstly, we selected and tested various primer sets to ensure the PCR efficiency of single locus. Then we optimized the annealing temperatures under which the multiple gene loci could be amplified successfully in a reaction mixture, without cross-reaction targets and primer dimers. Finally, three multiplex PCR systems were constructed for the 9 gene loci since it would be impossible to combine all the gene loci in one reaction mixture because some of them had different annealing temperatures. So based on the two-step library preparation, we used a total of three locus-specific PCRs, 
three cleanup reactions and one indexing PCR for 9 gene loci of one specimen, saving at least 6 cleanup reactions and 6 DNA quantities for each specimen, at a total cost of $<5$ USD due to reagent difference for amplification and library preparation. By Illumina Miseq sequencing, about 100000 raw reads were generated for each specimen in this study, costing about 6-8 USD to recover all loci. The sequence cost depends on the choice of sequence yield. Our multiplex PCR-based amplicon NGS sequencing makes it possible to generate nine locus dataset for a total sequence cost of $<5$ USD with sequence yield of 50000 raw reads per specimen. However, the taxonomic assignments between singleplex and multiplex PCR were not completely consistent. We found that both multiplex and singleplex PCR reactions generated some unique assignments. There was cross-identification between singleplex and multiplex PCR strategies. PCR bias affecting amplicon accuracy is inevitable, like DNA Polymerase, PCR cycle number, template concentration and annealing temperature, even for single amplicon PCR. Whatever, the multiplex PCR provides cost-efficient, quick and accurate amplicon NGS sequencing despite some bias. We will further focus on optimizing the multiplex PCR condition for assignment accuracy.

Finally we tested the efficiency of CMPM for monitoring diversity dynamics of plankton in Lake Tai. The assignments from the multiple primer sets were more than that from the morphological observations. The proportion of each taxonomic group assigned between April and August was generally consistent, but the total reads of samples from August was higher than that from April for the same sample size. These diversity variations suggest that the growth of phytoplankton changes with the time. The NMDS results indicated that the diversity of each phytoplankton phylum between April and August were significantly different, also for zooplankton and fungi. However, there was no much diversity difference for taxa among different regions of the lake in April or August, which is possibly because the bloom of Lake Tai in 2017 was extremely serious and happened in the maximum area of the lake unprecedentedly $\left(1403 \mathrm{~km}^{2}\right)$ (Zhu et al. 2019). The CCA analysis indicated that the samples from April and August were also separated clearly for all of phytoplankton, zooplankton and fungi. The temperature was shown as the remarkable environmental factor affecting the diversity variance among temperature. It has also been reported that the temperature stimulates cyanobacteria bloom in several ways like nitrogenase activity and growth rates and the plankton diversity changes with the seasons (Shi et al. 2017; Brauer et al. 2007; Paerl and Huisman, 2008; Jöhnk et al. 2008; Kosten et al. 2012; Su et al. 2012; Paerl et al. 2014). Our results are generally consistent with the previous reports. Thus our CMPM assay with new primer sets developed in this study would be more accurate and cost-effective for plankton monitoring and ecological research.

\section{Declarations}

\section{Funding}

The financial support from the China Postdoctoral Science Foundation (2014M561661, 2015T80558), National Natural Science Foundation of China (NSFC) (31600294) and the Fundamental Research Funds for the Central Universities (KJQN201742, Y0201600141) was gratefully acknowledged.

\section{Conflict of Interest}


The authors have no conflict of interest.

\section{Ethics approval}

Not applicable

\section{Consent to participate}

Not applicable

\section{Consent for publication}

Not applicable

\section{Availability of data and materials}

The NGS sequence data of all samples obtained in this study are deposited in NCBI. The SRA accession for the NGS sequences is PRJNA655206. The submission information for each sample mainly includes the reads sequence in fastq form, the sampling location, the sequence platform. These data will be public after the paper is published.

\section{Code availability}

Not applicable

\section{Acknowledgement}

Thanks the support of China Scholarship Council for studying in University of California, Berkeley, CA. Thanks very much Lydia Smith (University of California, Berkeley, CA) who helped much for the NGS library building, and thank the Evolutionary Genetics Lab (University of California, Berkeley, CA) for the lab work. Thanks for the help of Tyler linderoth, Joana L. Rocha and other members in Integrative biology department, Berkeley, CA, and the suggestions from Emily E. Curd (University of California - Los Angeles) about the reference library building, and coding and figure help from Meng Wang and Yuxiao Hua (Nanjing Agricultural University). The bioinformatics analysis of the project was supported by the Bioinformatics Center of Nanjing Agricultural University. Thanks very much the experimental platform support from the Laboratory of ecosystem ecology of Nanjing Agricultural University.

\section{References}

Alberdi, A., Aizpurua, O., Gilbert, MTP. \& Bohmann, K. Scrutinizing key steps for reliable metabarcoding of environmental samples. Methods Ecol Evol. 9, 134-147(2018).

Amaral-Zettler, L.A., Mccliment, E.A., Ducklow, H.W., \& Huse, S.M. A method for studying protistan diversity using massively parallel sequencing of $v 9$ hypervariable regions of small-subunit ribosomal rna genes. PLoS ONE 4, e6372 (2009). 
Bahram, M., Anslan, S., Hildebrand, F., Bork, P. \& Tedersoo, L. Newly designed 16S rDNA metabarcoding primers amplify diverse and novel archaeal taxa from the environment. Environ biol Rep. 9999(9999) (2018).

Baker, C. bakerccm/entrez_qiime: entrez_qiime v2.0. https ://doi.org/10.5281/zenodo.159607 (2016)

Baldwin, A. H., Jensen, K. \& Schoenfeldt, M. Warming increases plant biomass and reduces diversity across continents, latitudes, and species migration scenarios in experimental wetland communities. Global Change Biol. 20, 835-850 (2014).

Bartram, A.K., Lynch, M.D.J., Stearns, J.C., Moreno-Hagelsieb, G. \& Neufeld, J.D. Generation of multimillionsequence 16S rDNA gene libraries from complex bial communities by assembling paired-end illumina reads. Appl Environ biol. 77, 3846-3852 (2011).

Beng, K.C. \& Corlett, R.T. Applications of environmental DNA (eDNA) in ecology and conservation: opportunities, challenges and prospects. Biodivers Conservn. 29, 2089-2121 (2020).

Berdjeb, L. et al. Short-term dynamics and interactions of marine protist communities during the springsummer transition. Isme J, 2018.

Bolyen, E. et al. Reproducible, interactive, scalable and extensible biome data science using QIIME 2. Nat Biotechnol. 37, 852-857 (2019).

Bradley, I.M., Pinto, A.J. \& Guest, J.S. Design and Evaluation of Illumina MiSeq-Compatible, 18 S rDNA GeneSpecific Primers for Improved Characterization of Mixed Phototrophic Communities. Appl Environ biol. 82(19), 5878 (2016).

Bradley, I.M., Pinto, A.J., \& Guest, J.S. Design and evaluation of illumina miseq-compatible, 18S rRNA genespecific primers for improved characterization of mixed phototrophic communities. Appl Environ biol 82, 5878-5891 (2016).

Brauer, V. S. et al. Low temperature delays timing and enhances the cost of nitrogen fixation in the unicellular cyanobacterium Cyanothece. ISME J. 7, 2105-2115 (2013).

Bucklin, A., Lindeque, P. K., Rodriguez-Ezpeleta, N., Albaina, A., \& Lehtiniemi, M. Metabarcoding of marine zooplankton: Prospects, progress and pitfalls. J Plankton Res. 38, 393-400 (2016).

Burivalova, Z., Game, E.T. \& Butler, R.A. The sound of a tropical forest. Science 363, 28-29 (2019).

Camacho, C. et al. BLAST+: Architecture and applications. BMC Bioinformatics. 10, 421 (2009).

Claesson, M.J. et al. Comparison of two next-generation sequencing technologies for resolving highly complex biota composition using tandem variable 16S rDNA gene regions. Nucleic Acids Res. 38, e200 (2010).

Degnan, P.H. \& Ochman, H. Illumina-based analysis of bial community diversity. ISME J. 6, 183-194 (2012). 
Deorowicz, S., Debudaj-Grabysz, A., Gudys, A., FAMSA: Fast and accurate multiple sequence alignment of huge protein families. Sci Rep 6(1):33964 (2016).

Diez, B., Pedros-Alio, C., Marsh, T.L., \& Massana, R. Application of denaturing gradient gel electrophoresis (DGGE) to study the diversity of marine picoeukaryotic assemblages and comparison of DGGE with other molecular techniques. Appl Environ biol 67, 2942-2951(2001).

Drake, B. G. Rising sea level, temperature, and precipitation impact plant and ecosystem responses to elevated $\mathrm{CO} 2$ on a Chesapeake Bay wetland: review of a 28-year study. Global Change Biol. 20, 3329-3343 (2014).

Duan, H. et al. Distribution and incidence of algae blooms in Lake Tai. Aquat Sci. 77, 9-16 (2015).

Eisenstein, M., biology: making the best of PCR bias. Nat Methods. 15(5), 317 (2018).

Emily, E.C. et al. Anacapa Toolkit: An environMantel DNA toolkit for processing multilocus metabarcode datasets. Methods Ecol Evol, 10, 1469-1475 (2019).

Fadrosh, D.W. et al. An improved dual-indexing approach for multiplexed 16S rDNA gene sequencing on the Illumina MiSeq platform. biome 2, 6 (2014).

Ficetola, G.F. et al. An in silico approach for the evaluation of DNA barcodes. BMC Genom. 11, 434 (2010).

Franklin, T.W. et al. Using environmental DNA methods to improve winter surveys for rare carnivores: DNA from snow and improved noninvasive techniques. Biol Cons. 229, 50-58 (2019).

Gohl, D. M. et al. Systematic improvement of amplicon marker gene methods for increased accuracy in biome studies. Nat Biotechnol. 34, 942-949 (2016).

Henrik, K., Susan, R.K, Alexandra, Rueda., Athena, Lam. \& Rosemary, G.G. Scaling up DNA barcoding - Primer sets for simple and cost efficient arthropod systematics by multiplex PCR and Illumina amplicon sequencing. Methods Ecol Evol. (2018).

Hugerth, L.W. et al. DegePrime, a Program for Degenerate Primer Design for Broad-Taxonomic-Range PCR in bial Ecology Studies. Appl Environ biol. 80(16), 5116-23 (2014).

Illumina 16S metagenomic sequencing library preparation (Illumina Technical Note 15044223 Rev. A). Illumina http://support.illumina.com/content/dam/illuminasupport/documents/documentation/chemistry_documentation/16s/16s-metagenomic-library-prep-guide15044223-b.pdf (2013).

Jef, Huisman. et al. Cyanobacterial blooms. Nat Rev biol. 16, 471-483 (2018).

Jöhnk, K. D. et al. summer heatwaves promote blooms of harmful cyanobacteria. Glob Chang Biol. 14, 495512 (2008). 
Karst, S.M. et al. Retrieval of a million high-quality, full-length bial 16S and 18S rDNA gene sequences without primer bias. Nat Biotechnol. 36(2) (2018).

Knight, R. et al. Best practices for analysing biomes. Nat Rev biol. 16, 410-422 (2018).

Kosten, S. et al. Warmer climates boost cyanobacterial dominance in shallow lakes. Glob Chang Biol. 18, 118-126 (2012).

$\mathrm{Li}$, D. et al. Factors associated with blooms of cyanobacteria in a large shallow lake, China. Environ Sci Eur. 30(1), 27 (2018).

Lundberg, D.S., Yourstone, S., Mieczkowski, P., Jones, C.D. \& Dangl, J.L. Practical innovations for highthroughput amplicon sequencing. Nat Methods. 10, 999-1002 (2013).

Needham, D.M. \& Fuhrman, J.A. Pronounced daily succession of phytoplankton, archaea and bacteria following a spring bloom. Nat biology, 1(4), 16005 (2016).

Nübel, U., Garcia-Pichel., \& Muyzer, G. PCR primers to amplify 16S rRNA genes from cyanobacteria. Appl Environ biol 63, 3327-3332 (1997).

Nichols, R.V. et al. Minimizing polymerase biases in metabarcoding. Mol Ecol Resour. 18, 927-939 (2018).

Qin, B. et al. A drinking water crisis in Lake Tai, China: linkage to climatic variability and lake management. Environ Manag. 45, 105-112 (2010).

Oksanen, J. et al. vegan: Community Ecology Package R package version 2.5-5 (2019).

Outhwaite, C.L. et al. Complex long-term biodiversity change among invertebrates, bryophytes and lichens. Nat Ecol Evol 4, 384-392 (2020).

Paerl, H. W., Gardner, W. S., Mccarthy, M. J., Peierls, B. L. \& Wilhelm, S. W. Algae blooms: noteworthy nitrogen. Science 346, 175-175 (2014).

Paerl, H. W. \& Huisman, J. Blooms like it hot. Science 320, 57-58 (2008).

Pierre, T., Aurélie, B., Lucie, Z. \& Eric, C. EnvironMantel DNA For Biodiversity Research and Monitoring. OXFORD (2018).

Pruitt, K.D., Tatusova, T., \& Maglott, D.R. NCBI Reference Sequence (RefSeq): a curated non-redundant sequence database of genomes, transcripts and proteins. Nuleic Acids Res, 33, D501-D504 (2005).

Shelton, A.O. et al. Environmental DNA provides quantitative estimates of a threatened salmon species. Biol Conserv. 237, 383-391 (2019).

Sherwood, A.R., Presting, G.G., Sherwood, A.R, \& Presting, G.G. Universal primers amplify a 23S rDNA plastid marker in eukaryotic algae and cyanobacteria. J Phycology 43, 605-608(2007). 
Stoesser, G. et al. The EMBL Nucleotide Sequence Database. Nucleic Acids Res. 30(1), 21-26 (2002).

Takahara, T., Iwai, N., Yasumiba, K. \& Igawa, T. Comparison of the detection of 3 endangered frog species by eDNA and acoustic surveys across 3 seasons. Freshw Sci. 39, 18-27 (2020).

Ulrich, Nubel., PCR primer to amplify 16S rRNA genes from cyanobacteria, Appl Environ biol 63, 3327-3332 (1997).

Zhang, M. et al. Long-term dynamics and drivers of phytoplankton biomass in eutrophic Lake Tai. Sci Total Environ. 645, 876-886 (2018).

Zhu, W. et al. Analysis on the reasons for the large bloom area of Lake Taihu in 2017. J. Lake Sci. 31(3):621632(2019).

Zou, S. et al. How DNA barcoding can be more effective in algae identification: a case of cryptic diversity revelation in Scenedesmus (Chlorophyceae). Sci Rep, 6, 36822 (2016a).

Zou, S. et al. Combining and Comparing Coalescent, Distance and Character - Based Approaches for Barcoding algaes: A Test with Chlorella- Like Species (Chlorophyta). PLoS One, 11(4), e0153833 (2016b).

\section{Tables}


Table 1

Multiplex PCR systems and primers used and designed in this study.

\begin{tabular}{|c|c|c|c|c|c|}
\hline $\begin{array}{l}\text { PCR } \\
\text { system }\end{array}$ & Fragment & Primer Name & Sequence $\left(5^{\prime}-3^{\prime}\right)$ & Reference & $\begin{array}{l}\text { Fragment } \\
\text { size (bp) }\end{array}$ \\
\hline \multirow[t]{8}{*}{ A } & \multirow[t]{2}{*}{$16 \mathrm{~S}$} & CYA359F & GGGGAATYTTCCGCAATGGG & $\begin{array}{l}\text { Ulrich et } \\
\text { al., } 1997\end{array}$ & \multirow[t]{2}{*}{450} \\
\hline & & CYA781R & GACTACWGGGGTATCTAATCCCWTT & $\begin{array}{l}\text { Ulrich et } \\
\text { al., } 1997\end{array}$ & \\
\hline & \multirow[t]{2}{*}{ 18S-v1-3 } & Euklf & CTGGTTGATCCTGCCAG & $\begin{array}{l}\text { Diez rt } \\
\text { al., } 2001\end{array}$ & \multirow[t]{2}{*}{560} \\
\hline & & Euk516r & ACCAGACTTGCCCTCC & $\begin{array}{l}\text { Diez rt } \\
\text { al., } 2001\end{array}$ & \\
\hline & \multirow[t]{2}{*}{ 18S-v9 } & V8f & ATAACAGGTCTGTGATGCCCT & $\begin{array}{l}\text { Bradley } \\
\text { et al., } \\
2016\end{array}$ & \multirow[t]{2}{*}{375} \\
\hline & & $1510 \mathrm{R}$ & CCTTCYGCAGGTTCACCTAC & $\begin{array}{l}\text { Amaral- } \\
\text { Zettler, et } \\
\text { al., } 2009\end{array}$ & \\
\hline & \multirow[t]{2}{*}{$23 \mathrm{~S}$} & p23SrV_f1 & GGACAGAAAGACCCTATGAA & $\begin{array}{l}\text { Sherwood } \\
\text { et al., } \\
2007\end{array}$ & \multirow[t]{2}{*}{414} \\
\hline & & p23SrV_r1 & TCAGCCTGTTATCCCTAGAG & $\begin{array}{l}\text { Sherwood } \\
\text { et al., } \\
2007\end{array}$ & \\
\hline \multirow[t]{4}{*}{ B } & \multirow[t]{2}{*}{$28 \mathrm{~S}$} & 1FC1RF272F & GAGACCGATAGCRMACAAGT & $\begin{array}{l}\text { This } \\
\text { study }\end{array}$ & \multirow[t]{2}{*}{310} \\
\hline & & 1FC1RF593R & CTYGGTCCGTGTTTCWAGAC & $\begin{array}{l}\text { This } \\
\text { study }\end{array}$ & \\
\hline & \multirow[t]{2}{*}{$18 S-v 4-5$} & V4-384F & GYTGCAGTTAAAAMGCTCGT & $\begin{array}{l}\text { This } \\
\text { study }\end{array}$ & \multirow[t]{2}{*}{$500-520$} \\
\hline & & V4-879R & TTCAGNCTTGCGACCATACT & $\begin{array}{l}\text { This } \\
\text { study }\end{array}$ & \\
\hline
\end{tabular}




\begin{tabular}{|c|c|c|c|c|c|}
\hline $\begin{array}{l}\text { PCR } \\
\text { system }\end{array}$ & Fragment & Primer Name & Sequence $\left(5^{\prime}-3^{\prime}\right)$ & Reference & $\begin{array}{l}\text { Fragment } \\
\text { size (bp) }\end{array}$ \\
\hline \multirow[t]{6}{*}{$\mathrm{C}$} & \multirow[t]{2}{*}{ 16S-new } & 16SF174F & CGGTAAGACRGRGGATGCAA & $\begin{array}{l}\text { This } \\
\text { study }\end{array}$ & \multirow[t]{2}{*}{$550-700$} \\
\hline & & 16SR457R & CGACACGAGCTGACGACAGC & $\begin{array}{l}\text { This } \\
\text { study }\end{array}$ & \\
\hline & \multirow[t]{2}{*}{ rbcL-Chlo } & rbcLChloF24F & CGTATGACTCCWCAACCWGG & $\begin{array}{l}\text { This } \\
\text { study }\end{array}$ & \multirow[t]{2}{*}{400} \\
\hline & & rbcLChlo411R & GGTTTAATWGTACAWCCTAA & $\begin{array}{l}\text { This } \\
\text { study }\end{array}$ & \\
\hline & \multirow[t]{2}{*}{$r b c L$-Cry } & rbcLCryF $84 \mathrm{~F}$ & ATGTTCCGTAYGACWCCTCA & $\begin{array}{l}\text { This } \\
\text { study }\end{array}$ & \multirow[t]{2}{*}{350} \\
\hline & & rbcLCry405R & GCWGGACCTTGGWAAGTCTT & $\begin{array}{l}\text { This } \\
\text { study }\end{array}$ & \\
\hline
\end{tabular}

\section{Figures}

\section{Figure 1}

The workflow of NGS library preparation for the multiplex PCR-based eDNA profile.

\section{Figure 2}

The primer coverage for the newly designed genetic markers. Among the alignment position, the blue line represents the ratio of sequences used for primer selection among all alignment sequences. The orange dots represent the ratio of sequences where primer selected are included. The green dots represent the positions where the conserved primers were finally used. 

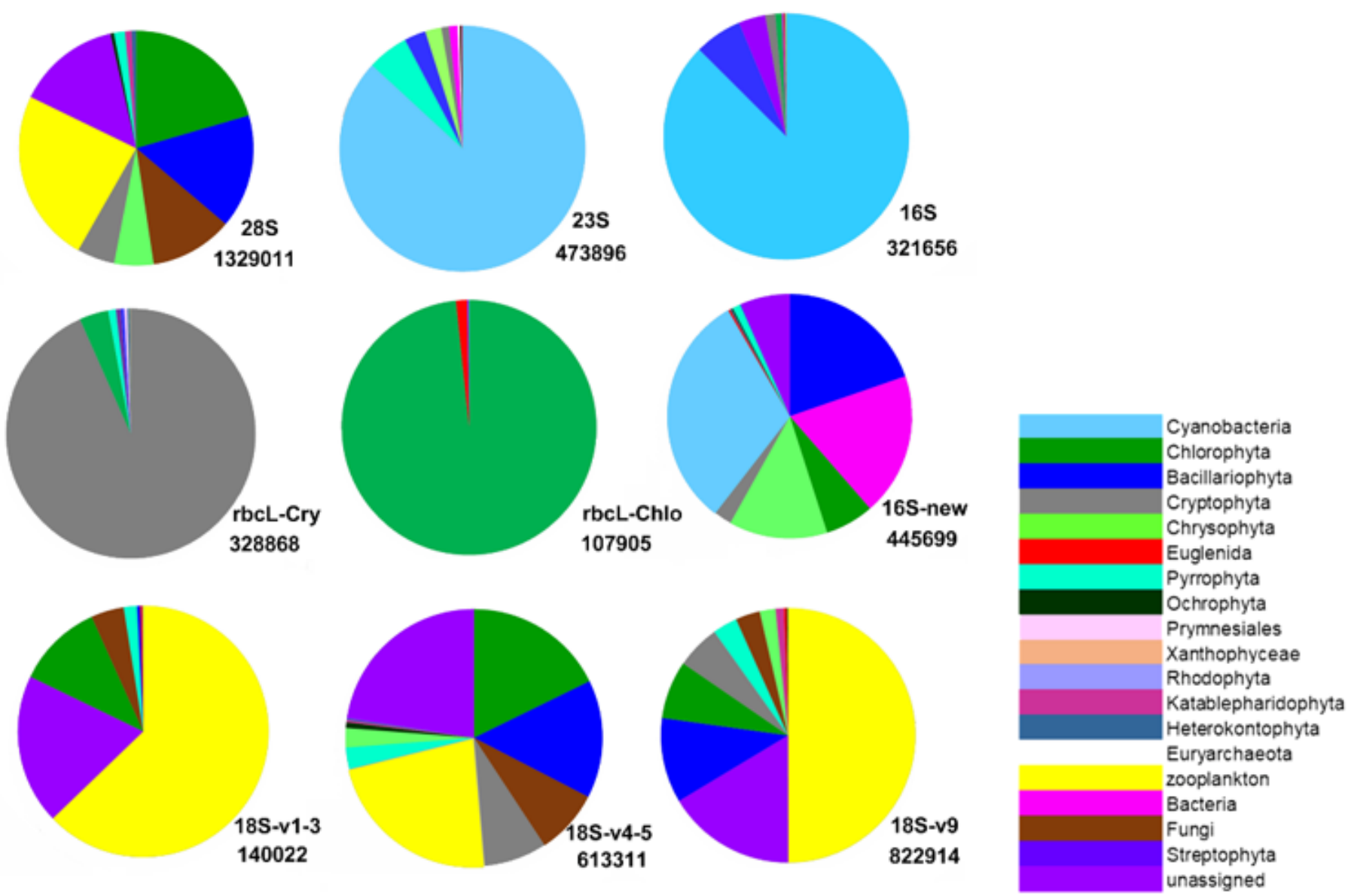

\section{Figure 3}

The proportion of various taxonomic groups assigned from each gene barcode, where each phytoplankton phylum was analyzed independently.

\section{Figure 4}

Venn diagram for taxonomic difference among all genes for Bacillariophyta, Chlorophyta, Cyanobacteria and Zooplankton respectively. Set size in black bar (lower left) represents the total assignments from each gene beside it. Each dark spot (lower right) is consistent with each gene left. The intersection size in top color bar represents the amount of unique assignments from single gene (single dark spot) and the identical assignments from several genes (the vertical line stringing the dark spots). The top red, green and purple bars represent species, genus and family level respectively.

\section{Figure 5}


The difference of taxonomic assignments among all genes and microscope observations at species level for each phytoplankton phylum, from total of the reference samples. The annotation for the figure is the same with that in Figure 5.

\section{Figure 6}

The assignment rate in species, genus and family level from each genetic marker for Cyanobacteria, Bacillariophyta, Chlorophyta, Cryptophyta and Zooplankton respectively. This assignment rate means the ratio of taxa in species, genera and families level among all the final assignments for each marker.

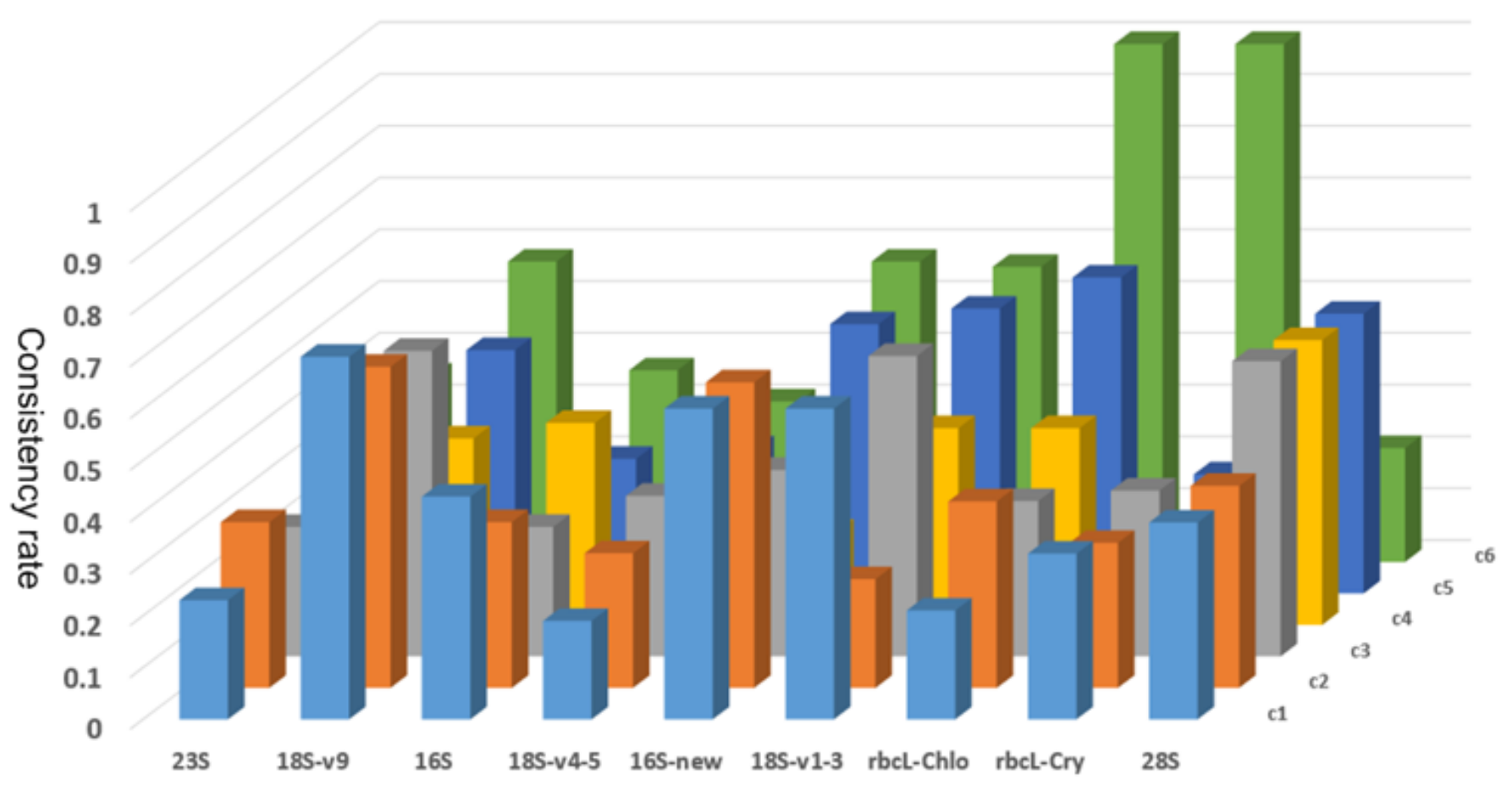

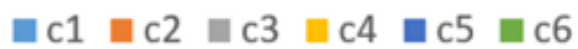

\section{Figure 7}

The assignment consistency of single and multiplex PCR in species level, which was performed by observing the ratio of their shared assignments to their total assignments in c1-c6 samples for each genetic marker.

\section{Figure 8}

The reads proportion of various taxonomic groups assigned for April and August respectively (A). The heatmap presenting abundance difference of top 30 phytoplankton species (B) and top 30 genera (C) among 
different regions of the lake for April and August respectively. The phylum name (left) and order number for abundance (right) is besides species name. The color represents the degree of the abundance, where the red and blue color indicates higher and lower abundance respectively.

\section{Figure 9}

NMDS plots showing diversity dissimilarities among all samples of different regions of the whole lake. The R2 values were shown for each plot. For Bacillariophyta, Chlorophyta, Cryptophyta, fungi and zooplankton, the reference and non-reference samples were separated clearly due to their different assignments by different primer sets, where the "reference" and "non-reference" were indicated besides the samples. For Cyanobactetia, Chrysophyta, Euglenida and Pyrrophyta, the reference and non-reference samples were not separated clearly. Here the mock means "reference".

\section{Figure 10}

Canonical correspondence analysis (CCA) ordination diagram for correlations of diversity dynamics and environmental factors, where the non-reference samples identified by microscope observations samples amplified from PCR system A in Table 1 (methods and materials) were included. The length of the vector is proportional to its importance, and the angle between two vectors reflects the degree of correlation between variables. WD: Water temperature; TN: Total nitrogen; TP: Total phosphorus; NH4+-N: Ammonia nitrogen; NO3-N: Nitrate nitrogen.

\section{Supplementary Files}

This is a list of supplementary files associated with this preprint. Click to download.

- TableS1.xlsx

- Tables2.xIsx

- FigureS1.tif

- Figures2.tif

- Figures3.tif

- FigureS4.tif

- Figures5.tif

- Figures6.tif

- Figures7.tif

- Figures8.tif 